\title{
Xenon Protects against Blast-Induced Traumatic Brain Injury in an In Vitro Model
}

\author{
Rita Campos-Pires, ${ }^{1,2}$ Mariia Koziakova,, ${ }^{1,2}$ Amina Yonis, ${ }^{1}$ Ashni Pau, ${ }^{1}$ Warren Macdonald, ${ }^{2,3}$ Katie Harris, \\ Christopher J. Edge, ${ }^{4,5}$ Nicholas P. Franks, ${ }^{4}$ Peter F. Mahoney, and Robert Dickinson ${ }^{1,2}$
}

\begin{abstract}
The aim of this study was to evaluate the neuroprotective efficacy of the inert gas xenon as a treatment for patients with blast-induced traumatic brain injury in an in vitro laboratory model. We developed a novel blast traumatic brain injury model using C57BL/6N mouse organotypic hippocampal brain-slice cultures exposed to a single shockwave, with the resulting injury quantified using propidium iodide fluorescence. A shock tube blast generator was used to simulate open field explosive blast shockwaves, modeled by the Friedlander waveform. Exposure to blast shockwave resulted in significant $(p<0.01)$ injury that increased with peak-overpressure and impulse of the shockwave, and which exhibited a secondary injury development up to $72 \mathrm{~h}$ after trauma. Blast-induced propidium iodide fluorescence overlapped with cleaved caspase- 3 immunofluorescence, indicating that shock-wave-induced cell death involves apoptosis. Xenon (50\% $\mathrm{atm})$ applied $1 \mathrm{~h}$ after blast exposure reduced injury $24 \mathrm{~h}(p<0.01), 48 \mathrm{~h}(p<0.05)$, and $72 \mathrm{~h}(p<0.001)$ later, compared with untreated control injury. Xenon-treated injured slices were not significantly different from uninjured sham slices at $24 \mathrm{~h}$ and $72 \mathrm{~h}$. We demonstrate for the first time that xenon treatment after blast traumatic brain injury reduces initial injury and prevents subsequent injury development in vitro. Our findings support the idea that xenon may be a potential first-line treatment for those with blast-induced traumatic brain injury.
\end{abstract}

Keywords: blast-induced neurotrauma; blast traumatic brain injury; neuroprotection; primary blast injury; TBI; xenon

\section{Introduction}

$\mathbf{T}$ RAUMATIC BRAIN INJURY (TBI) is a leading cause of death and disability in both military and civilian populations, ${ }^{1-3}$ but there are, as yet, no effective treatments targeting injury development. Blast-TBI has been called a "signature injury" of recent military operations in Iraq and Afghanistan. ${ }^{2,3}$ While blunt and penetrating TBI injury have been studied extensively, blast-TBI is much less well understood but is being recognized now as having a unique pathophysiology. ${ }^{4}$ The prevalence of blast-injury in recent returning veteran populations ${ }^{5,6}$ has prompted research into blast-TBI. Studies in rodents, swine, and other mammals have shown that exposure to blast-overpressure can result in a variety of behavioral impairments and neuropathological abnormalities. ${ }^{7-9}$

Despite the increasing research focus on blast-TBI pathophysiology, there are no clinically proven treatments to prevent or limit ongoing brain injury after blast-TBI. In addition, to date there have been few pre-clinical studies evaluating potential treatments or reporting improved outcomes after blast-TBI. ${ }^{8,10,11}$ There is an urgent need for treatments aimed at mitigating the neurological and cognitive deficits caused by blast-TBI and promoting a more rapid and complete recovery.

Xenon is a nonflammable inert gas that has been used as a general anesthetic since the 1950s. ${ }^{12,13}$ Xenon has been shown to be neuroprotective in a variety of in vitro and in vivo models of ischemia and stroke. ${ }^{14-23}$ Xenon is a pleiotropic drug known to act via a number of targets implicated in secondary injury development including inhibition of N-methyl-D-aspartate receptors, ${ }^{24-26}$ activation of potassium channels, ${ }^{27,28}$ and anti-apoptotic action. ${ }^{29}$ Xenon has a number of unique advantages including not being metabolized and rapidly crossing the blood-brain barrier facilitating a rapid onset and offset of action, within minutes. A recent clinical trial of xenon for ischemic brain injury in patients with

\footnotetext{
${ }^{1}$ Anaesthetics, Pain Medicine and Intensive Care Section, Department of Surgery and Cancer, ${ }^{2}$ Royal British Legion Centre for Blast Injury Studies, Department of Bioengineering, ${ }^{3}$ Department of Bioengineering, ${ }^{4}$ Department of Life Sciences, Imperial College London, London, United Kingdom.

${ }^{5}$ Department of Anaesthetics, Royal Berkshire Hospital NHS Foundation Trust, Reading, United Kingdom.

${ }^{6}$ Royal Centre for Defence Medicine, Medical Directorate Joint Force Command, ICT Centre, Birmingham, United Kingdom.

(c) Rita Campos-Pires et al., 2018; Published by Mary Ann Liebert, Inc. This Open Access article is distributed under the terms of the Creative Commons License (http://creativecommons.org/licenses/by/4.0), which permits unrestricted use, distribution, and reproduction in any medium, provided the original work is properly credited.
} 
out-of-hospital cardiac arrest showed that xenon administered within $6 \mathrm{~h}$ after injury reduces cerebral white matter damage. ${ }^{30}$

We have shown recently that xenon mitigates brain injury progression and improves long-term outcome in blunt-TBI in rodents. ${ }^{31}$ Here we describe a novel in vitro model of blast-TBI, which we utilize to test the hypothesis that xenon treatment can prevent or limit brain injury after blast-wave exposure.

\section{Methods}

Experiments complied with the United Kingdom (UK) Animals Scientific Procedures Act (1986) and were approved by the Animal Welfare and Ethical Review Body of Imperial College London. Unless otherwise stated, reagents were purchased from Sigma Aldrich (Dorset, UK).

\section{The shock tube blast generator}

A shock tube was used to generate controlled overpressure waves that model real-life free-field explosions with a Friedlander-type waveform. ${ }^{32}$ The shock tube (Fig. 1A) is a $3.8 \mathrm{~m}$ long horizontal stainless steel tube, with three $1.22 \mathrm{~m}$ long sections, with internal diameter of $0.059 \mathrm{~m}$ and external diameter of $0.073 \mathrm{~m}$. The first section, $1.22 \mathrm{~m}$ long, is the high pressure driver section, separated from the low pressure (1 atm) section, $2.44 \mathrm{~m}$ long, by a double breech assembly, $0.08 \mathrm{~m}$ long. The double breech allows one or two Mylar ${ }^{\circledR}$ polyester diaphragms (RS Components, Northants, UK) to be clamped between the high pressure driver section and the driven section with gas tight seals provided by nitrile O-rings (Fig. 1A inset). The effective driver volume can be adjusted by including one or more cylindrical polyethylene "blanking" sections inside the driver section.

For these in vitro experiments, we used a $15 \%$ driver volume in the single and double diaphragm configuration to generate shockwaves with peak overpressures of $55 \mathrm{kPa}$ and $50 \mathrm{kPa}$, respectively. The driver section was pressurized with compressed air (BOC,
Guildford, UK). Burst pressure is determined by the diaphragm material and thickness and in the double diaphragm configuration is triggered by venting the breech section through a fast-acting servovalve. Two ultrafast piezoelectric high dynamic pressure transducers (2300 V1, Dytran Instruments, Chatsworth, CA) were mounted radially on the shock tube. Sensor 1, mounted in the middle of the driven section, was used for triggering data acquisition; sensor 2, mounted at the end of the driven section, was used for measurement of shockwave peak overpressure and duration (Fig. 1). The pressure transducers were connected to a current source power unit (model 4103C, Dytran Instruments) and output signals recorded on a high bandwidth oscilloscope (model DPO4104B, Tektronix Inc, Beaverton, OR).

Shockwave data were acquired at a sampling rate of $50 \mathrm{MHz}$ over $20 \mathrm{msec}\left(1 \times 10^{6} \mathrm{samples} / \mathrm{wave}\right)$. Voltage signals were saved on digital storage media and analyzed offline on a computer using MATLAB software (Release 2015a, MathWorks, Natick, MA) and the appropriate calibration factor for each pressure transducer provided by the manufacturer.

\section{In vitro slice cultures}

Organotypic hippocampal slice cultures (OHSCs) were prepared from postnatal day five to seven C57BL/6N mouse pups under aseptic conditions using the interface method described by Stoppini and associates ${ }^{33}$ with some modifications. ${ }^{34-36}$ Slices on tissue culture inserts (Millicell-CM, Millipore, Carrigtwohill, Ireland) were cultured at $37^{\circ} \mathrm{C}$ with $5 \% \mathrm{CO}_{2}$ in air in a humidified incubator (BB6220, Heraeus, Germany) for 12-14 days. The growth medium was changed on the first day of culture and every two to three days thereafter.

\section{Preparation of OHSCs for in vitro blast-TBI}

After 12-14 days in culture, the tissue culture inserts were transferred to six-well culture plates (Nunc, Roskilde, Denmark)
A

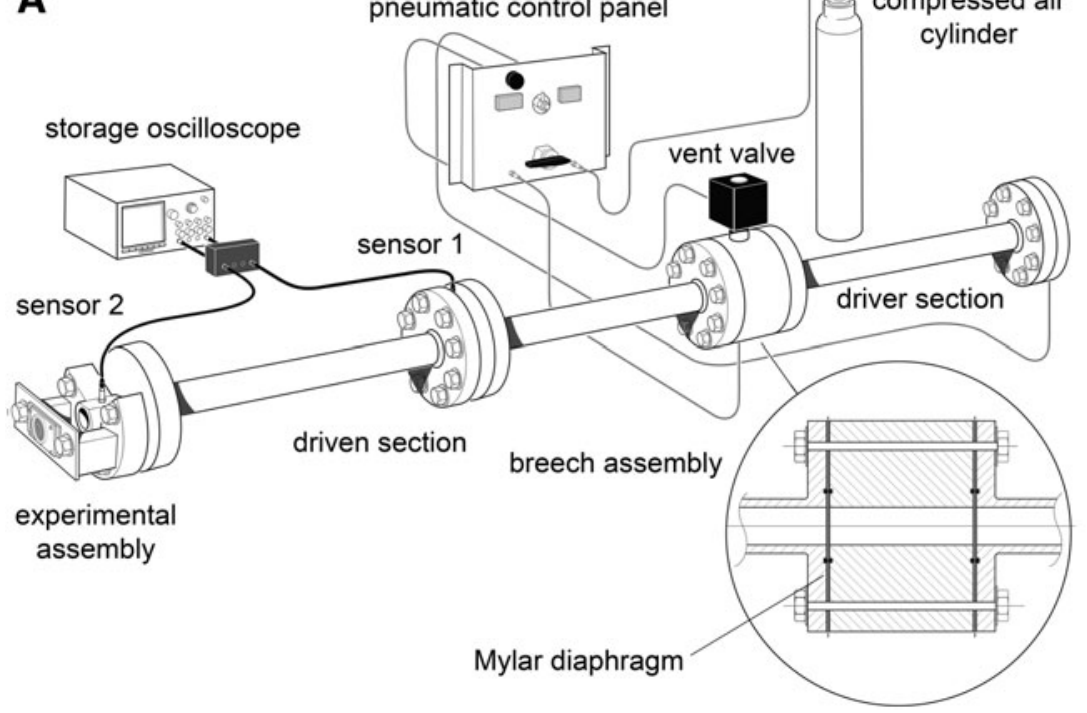

B

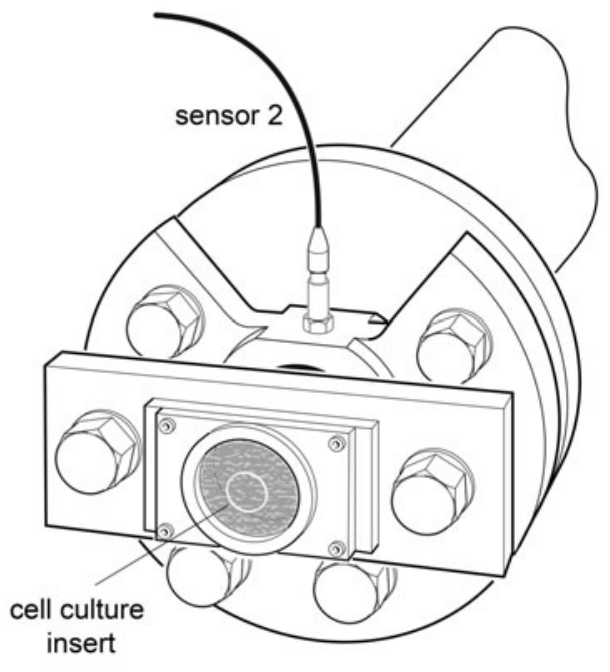

FIG. 1. (A) Schematic drawing of the shock tube used for these studies. The driver section is filled with compressed air via a control panel with control valves and pressure gauges allowing separate control of the pressure in the breech and the driver tube. A solenoidcontrolled vent valve allows the pressure in the breech to be rapidly vented, triggering diaphragm rupture in the double-breech configuration. The breech (inset) can be used in single-diaphragm or double-diaphragm configuration. The driven section (left of the breech) has two ultrafast rise-time dynamic pressure transducers, mounted radially, halfway along (sensor 1) and at the distal end (sensor 2). These sensors are connected to a high bandwidth digital storage oscilloscope. (B) Detail of the shock tube assembly used in these in vitro experiments. The Millicell culture insert (small grey circle) with organotypic slices is inside a polythene bag containing experimental medium. The polythene bag is clamped all around a circular aperture in front of the shock-tube distal flange. 
containing pre-warmed $\left(37^{\circ} \mathrm{C}\right)$ serum-free "experimental medium" with propidium iodide (PI) (75\% minimum essential medium Eagle; $25 \%$ Hank balanced salt solution; $5 \mathrm{mg} / \mathrm{mL}$ D-glucose; $2 \mathrm{mM} \mathrm{L-glutamine;} 1 \%$ antibiotic-antimycotic solution; $10 \mathrm{mM}$ HEPES; $4.5 \mu \mathrm{M}$ PI; $\mathrm{pH}$ titrated to 7.2 ). At $1 \mathrm{~h}$ after transfer to experimental medium, the slices were imaged (see Quantifying cell injury) to assess baseline slice health before injury and to ensure that all slices were equivalent at the start of the procedure (and before allocating to experimental groups). A small number of slices exhibited areas of dense staining at this stage (likely because of mechanical damage occurring during slice preparation) and were excluded from further analysis.

Immediately after baseline imaging, individual tissue culture inserts were sealed carefully in sterile polyethylene sample bags (Twirl'em ${ }^{\circledR} 3$ " $\times 5$ ", Fisher Scientific, Loughborough, UK) using aseptic conditions. Each bag was pre-filled with warmed $\left(37^{\circ} \mathrm{C}\right)$ experimental medium that had been saturated by bubbling with $95 \% \mathrm{O}_{2} ; 5 \% \mathrm{CO}_{2}$ for $45 \mathrm{~min}$. Gas bubbles were carefully excluded on sealing sample bags. We determined whether slice submersion affected slices and found no significant difference between slices that had been maintained without submersion and those submerged (data not shown).

\section{In vitro blast injury procedure}

At $1 \mathrm{~h}$ after submersion of the tissue culture insert, each sample bag was assigned randomly to either blast or sham group and clamped symmetrically all round in a vertical position in front of the shock-tube outlet using a custom-made stainless steel assembly (Fig. 1B) with the inserts positioned perpendicular to the axis of the shock tube with the OHSCs facing the shock tube. The OHSCs in the blast groups then were exposed immediately to a single shockwave (Fig. 2B). After blast-injury or sham procedure, each sample bag was placed in a thermo-regulated box $\left(37^{\circ} \mathrm{C}\right)$ before OHSCs were returned to air-liquid interface conditions.

Our objective was to develop a model that was sensitive to injury induced by a primary blast wave. Because of the sensitivity of our measurement of cell death, and in common with other in vitro brain injury models, ${ }^{35,36}$ there is a degree of injury in the sham slices. For this reason, we ensured that every experiment included a sham group, and we randomly allocated slices to sham and blast groups. We took care to ensure that sham slices were treated identically to the slices exposed to a shockwave (sealed in the sample bags and suspended on the stainless steel rig for an equivalent period), but the shock tube was not fired. The level of injury in the sham slices at $24 \mathrm{~h}$ and $48 \mathrm{~h}$ was similar to that observed in our in vitro blunt TBI model, ${ }^{36}$ but at $72 \mathrm{~h}$ was greater than the blunt TBI model, likely reflecting the greater slice manipulation in the blast model. The injury in the blast-exposed slices was significantly $(p<0.01)$ greater than sham slices at all time points after blast.

In some of the earlier experiments, to be able to distinguish between the different magnitudes of blast $(50 \mathrm{kPa} ; 55 \mathrm{kPa})$ and to assess how injury developed after both blast intensities, we had larger group sizes ( $\sim 30-50$ slices). In the xenon neuroprotection experiments, we chose the higher blast intensity $(55 \mathrm{kPa})$, and in these experiments, smaller group sizes $(\sim 12)$ were used for the
A

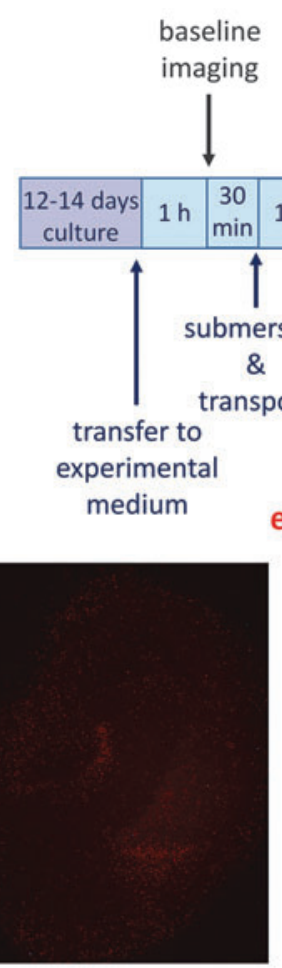

sham

\section{$24 \mathrm{~h}$}

imaging

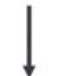

gas treatment (chamber)
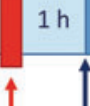

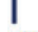

air-interface

\&

start treatment

blast

wave

exposure

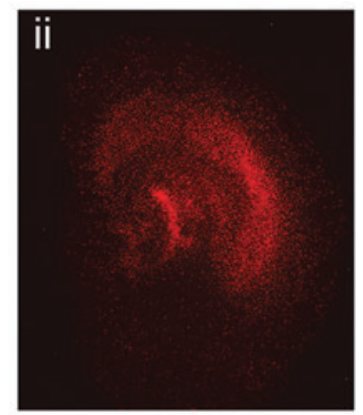

blast

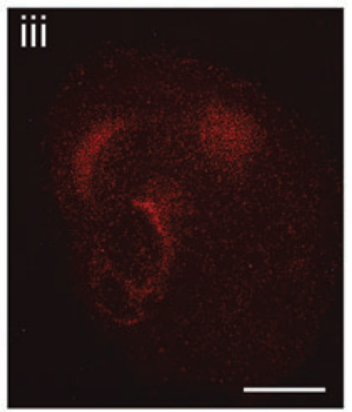

blast + xenon

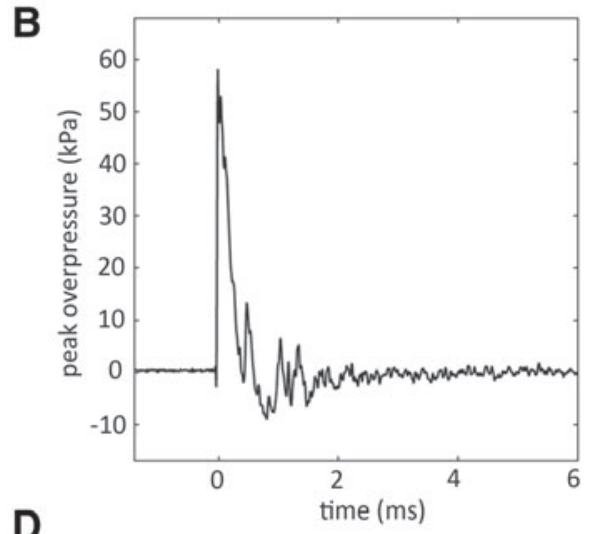

D

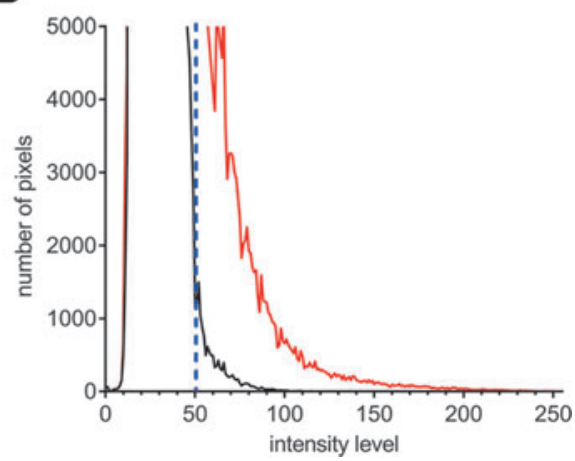

FIG. 2. (A) Experimental timeline. (B) A representative shockwave obtained using single 23- $\mu$ m Mylar ${ }^{\circledR}$ diaphragm, peak overpressure $55 \mathrm{kPa}$, positive wave duration $0.4 \mathrm{msec}$, and impulse $10.3 \mathrm{kPa} \bullet \mathrm{msec}$. Shockwave data were obtained from a pressure transducer mounted radially on the distal flange of the driven section. (C) Images showing typical (i) uninjured sham slice, (ii) blastTBI exposed slice, and (iii) xenon-treated blast-TBI slice, at $72 \mathrm{~h}$ after injury. The red propidium iodide fluorescence is used as a marker of dead or dying cells. Scale bar $=500 \mu \mathrm{m}$. (D) For each slice, an image intensity histogram was produced and injury quantified by counting the number of pixels above a threshold of 50 in the intensity histograms (dashed vertical line). The lines shown indicate the intensity histograms of images of a typical sham slice (black line) and a typical blast-injured slice (red line) at $72 \mathrm{~h}$ after the blast or sham procedure. 
injury and treatment groups; because we included a sham group per condition in every experiment, the sham group was larger $(\sim 23)$.

\section{Experimental gas treatment}

After the blast-TBI procedure, inserts were carefully removed from the sample bags inside a laminar flow tissue culture hood and transferred to six-well culture plates with pre-warmed $\left(37^{\circ} \mathrm{C}\right)$ experimental medium. The six-well plate was transferred back to either the humidified incubator $\left(37^{\circ} \mathrm{C}, 5 \% \mathrm{CO}_{2}\right.$ in air) or, for the gas treatment experiments, plates were randomly assigned to either xenon or control gas (helium) groups and transferred to one of two small custom-made hyperbaric chambers containing a high-speed fan for rapid gas mixing, housed in an incubator set at $37^{\circ} \mathrm{C} .^{34,35}$ The chamber (volume $0.925 \mathrm{~L}$ ) was flushed with humidified control gas $\left(20 \% \mathrm{O}_{2} ; 75 \% \mathrm{~N}_{2} ; 5 \% \mathrm{CO}_{2}\right)$ for $5 \mathrm{~min}$ at $5 \mathrm{~L} / \mathrm{min}^{36}$ The chamber was then sealed and an additional $50 \%$ atm of helium or xenon was added (the total final pressure inside the chamber was $1.5 \mathrm{~atm}$ ). The partial pressures of $\mathrm{O}_{2}$ and $\mathrm{CO}_{2}$ were fixed at $0.2 \mathrm{~atm}$ and $0.05 \mathrm{~atm}$, respectively.

Six-well plates were removed from the chamber at $24 \mathrm{~h}, 48 \mathrm{~h}$, and $72 \mathrm{~h}$ for imaging (Fig. 2A). For quantification of injury, slices in six well plates were allocated a well identifier $\mathrm{A}-\mathrm{F}$ and a slice number (e.g., 1-4). Imaging was performed and data files stored with reference to these identifiers until the final analysis. After imaging, plates were transferred back to the chamber and the appropriate gas mixture was reestablished.

\section{Quantifying cell injury}

The PI only enters cells with compromised cellular membranes and becomes fluorescent after binding to nucleic acids allowing quantification of cell injury. ${ }^{37-39}$ An epifluorescence microscope (Nikon Eclipse 80i, Kingston upon Thames, UK) with a low power objective (Nikon Plan UW $2 \times$ magnification, NA 0.06) was used to visualize PI fluorescence (Fig. 2C). A digital camera (Micropublisher 3.3 RTC) and image capture software (QCapturePro, Qimaging Inc., Surrey, British Columbia, Canada), were used as described previously. ${ }^{36}$ Image intensity analysis of the red channel was performed using ImageJ software, ${ }^{40}$ with the distribution of intensities plotted as a histogram over 256 intensity levels. Slices under uninjured conditions exhibited a well-defined peak in the intensity distribution, which fell rapidly to zero. As a measure of injury caused by the shockwave exposure, the number of pixels above a threshold of 50 was integrated (Fig. 2D). ${ }^{36}$

\section{Immunofluorescence}

At the end of the experiment, slices on Millicell inserts were washed with warmed $\left(37^{\circ} \mathrm{C}\right)$ phosphate-buffered saline (PBS) followed by fixation for $1 \mathrm{~h}$ with $4 \%$ paraformaldehyde (ThermoFisher, Loughborough, UK) in PBS. Slices were detached carefully from inserts and mounted on glass slides. Slices were blocked for 90 min with $10 \%$ goat serum in PBS, incubated overnight at $4^{\circ} \mathrm{C}$ with cleaved caspase-3 primary antibody (9661S, Cell Signaling Technology, Leiden, Netherlands, dilution 1:200) followed by $1 \mathrm{~h}$ incubation at room temperature with Alexa Fluor ${ }^{\circledR} 488$ goat antirabbit secondary antibody (A-11008, Life Technologies, Paisley, UK, dilution 1:500), with $3 \times$ washes with PBS $0.3 \%$ Triton X 100 between each step. Slices were imaged using a Nikon Eclipse 80i microscope with $\mathrm{a} \times 100$ objective (Nikon Plan Fluor, NA 1.3 oil, WD 0.20).

\section{Statistics}

Results are expressed as mean \pm standard error of the mean Significance was assessed using a two-way repeated measures analysis of variance with Holm-Sidak post hoc test. Factor 1 was treatment (sham, xenon, control), and factor 2 was time before or after the injury $(-1 \mathrm{~h}, 24 \mathrm{~h}, 48 \mathrm{~h}$, and $72 \mathrm{~h}$ ), where factor 1 was the repeated factor. Statistical tests were implemented using GraphPad Prism (GraphPad, La Jolla, CA).

\section{Results}

\section{In vitro blast-TBI model}

To determine the optimum level of traumatic injury for neuroprotection experiments, we determined how the peak overpressure (POP) of Friedlander waveform shockwaves (Fig. 2B) affected injury magnitude and development. The OHSCs exposed to blast-injury exhibited increased PI fluorescence, compared with identically treated sham slices (Fig. 2C i, ii). In all experiments, to ensure that slices allocated to each experimental group were the same at the start of the procedure, we measured the PI fluorescence $1 \mathrm{~h}$ before starting the blast injury protocol. The baseline fluorescence at $\mathrm{t}=-1 \mathrm{~h}$ was very low (typically $2 \%$ of control injury at $72 \mathrm{~h}$ ) and was not significantly different between any of the groups (Fig. 3A,C,D). We quantified injury development as a function of shockwave peak overpressure, at $24 \mathrm{~h}, 48 \mathrm{~h}$, and $72 \mathrm{~h}$ after injury (Fig. 3A).

Shockwaves with POPs of $50 \mathrm{kPa}$ (duration $0.4 \mathrm{msec}$; impulse $8.8 \mathrm{kPa} \bullet \mathrm{msec}$ ) and $55 \mathrm{kPa}$ (duration $0.4 \mathrm{msec}$; impulse $10.3 \mathrm{kPa} \bullet \mathrm{msec}$ ) resulted in injury that developed up to $72 \mathrm{~h}$ after blast exposure. Injury magnitude increased with shockwave intensity; the $55 \mathrm{kPa}$ POP shockwave produced a significantly $(p<0.05)$ larger injury than the $50 \mathrm{kPa}$ POP shockwave, at $24 \mathrm{~h}$, $48 \mathrm{~h}$, and $72 \mathrm{~h}$ after blast-injury (Fig. 3A). Shockwaves of $55 \mathrm{kPa}$ POP were chosen as our standard injury because they produced a consistent and reproducible traumatic injury that developed significantly $(p<0.0001)$ over $72 \mathrm{~h}$, compared with uninjured slices.

Blast-TBI-induced cell death involves apoptotic pathways as shown by an increase in cleaved caspase- 3 after blast and strong colocalization with the PI staining (Fig. 3B). In contrast, in sham slices, not exposed to blast, while there was a low level of cell death indicated by PI staining, this was not associated with caspase activation (Fig. 3B).

\section{Lack of effect of helium}

Because the xenon experiments would use 50\% atm xenon, we determined whether pressure per se would affect the injury. The effect of $50 \%$ atm helium on sham-treated and blast-injured slices was investigated (Fig. 3C). Helium was chosen because we have shown in other in vitro brain injury models that helium at these low pressures does not affect OHSCs. ${ }^{35,36}$ Adding 50\% atm helium had no significant effect $(p>0.87)$ on either the sham or blast-injured slices at any time point. Nevertheless, in all the experiments with xenon, we used $50 \%$ atm helium as the control.

\section{Neuroprotection by xenon}

We evaluated the neuroprotective efficacy of treatment with $50 \%$ atm xenon starting $1 \mathrm{~h}$ after blast-TBI. Xenon had a protective effect against blast trauma at all time points (Fig. 3D). Injury in the xenon-treated slices was reduced by $47 \pm 12 \%(p<0.01)$ compared with the untreated injured slices $24 \mathrm{~h}$ after injury. In the xenontreated group at $48 \mathrm{~h}$ after blast exposure, injury was reduced by $31 \pm 7 \%(p<0.05)$. At $72 \mathrm{~h}$ after injury, the injury was reduced by $39 \pm 7 \%(p<0.001)$ in the xenon-treated group. The blast-injured slices treated with xenon were not significantly different from the uninjured sham slices at $24 \mathrm{~h}$ and $72 \mathrm{~h}$ after injury. 
A Injury development after in vitro blast TBI depends on intensity of blast wave

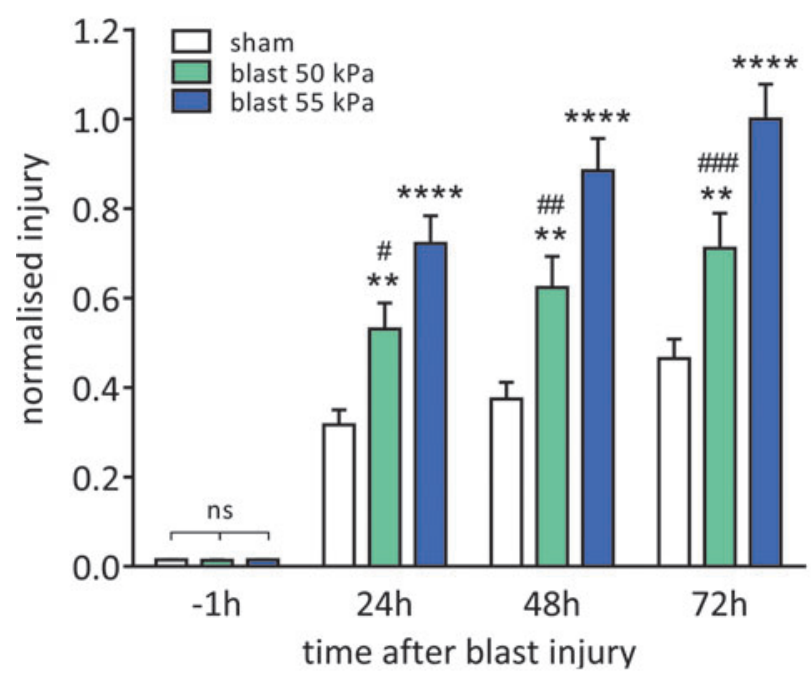

C Helium has no effect on sham or blast slices

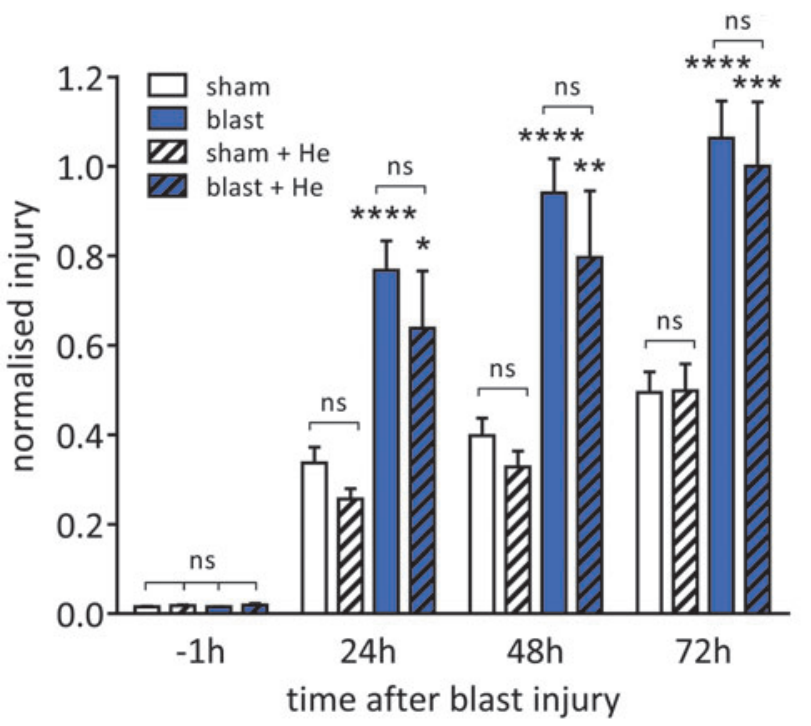

B Blast TBI in vitro results in caspase 3 activation

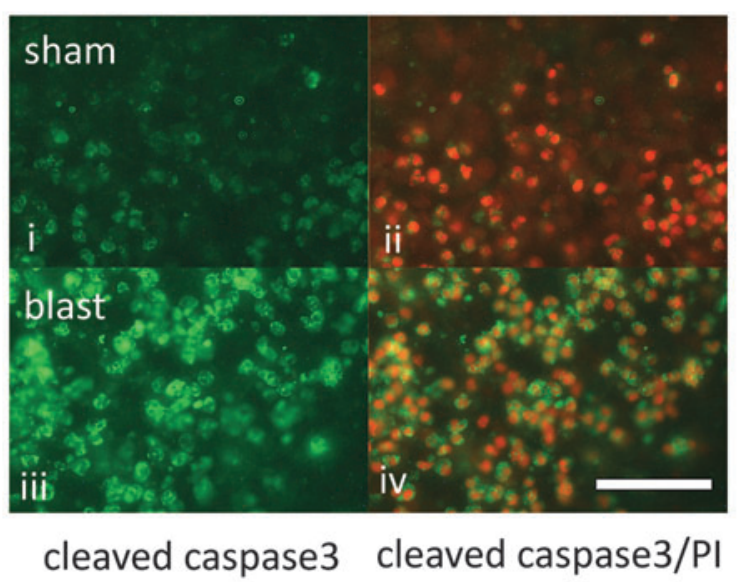

D Xenon protects against blast TBI in vitro

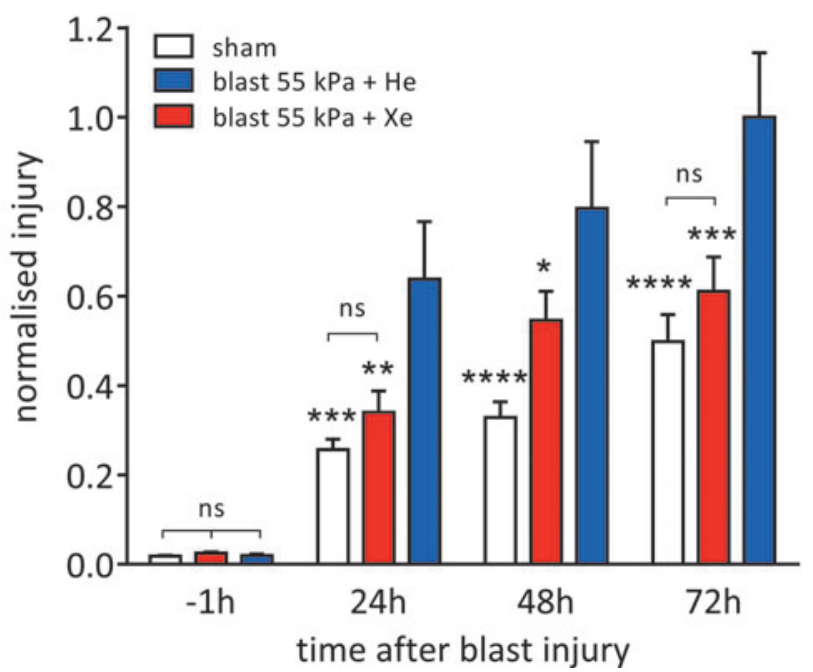

FIG. 3. A) The magnitude of the developing injury is dependent on the intensity of the shockwave. Shockwaves with peak overpressures of $50 \mathrm{kPa}(8.8 \mathrm{kPa} \bullet \mathrm{msec}$ impulse) (green bars) and $55 \mathrm{kPa}(10.3 \mathrm{kPa} \bullet \mathrm{msec}$ impulse) (blue bars), resulted in a significant injury compared with uninjured sham-slices (white bars). Data have been normalized to control blast $55 \mathrm{kPa}$ at $72 \mathrm{~h}$ after injury. Bars represent mean values and error bars are standard errors $(n=48$, sham; $n=30$, blast $50 \mathrm{kPa} ; n=51$, blast $55 \mathrm{kPa})$. $* * p<0.01, * * * * p<0.0001$

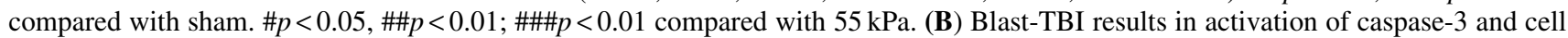
death. (i) Uninjured sham stained for cleaved caspase-3 (green); (ii) overlay of cleaved caspase-3 (green) and propidium iodide (PI) (red) in uninjured sham slice; (iii) slice exposed to $55 \mathrm{kPa}$ shockwave showing increase in cleaved caspase-3 staining (green); (iv) overlay of cleaved caspase-3 (green) and PI (red) in $55 \mathrm{kPa}$ shockwave exposed slice showing co-localization of both makers. Scale bar $=50 \mu \mathrm{m}$. (C) Helium (50\% atm) has no effect on the sham or $55 \mathrm{kPa}$ blast-injured slices. Sham-treated slices are shown as white bars (no helium) or white hatched bars (helium), and blast-injured slices are shown as blue bars (no helium) or blue hatched bars (helium). Data have been normalized to control blast $+50 \%$ atm helium at $72 \mathrm{~h}$ after injury. Bars represent mean values, and error bars are standard errors $(n=48$, sham; $n=51$, blast; $n=22$, sham $+50 \%$ atm helium; $n=12$, blast $+50 \%$ atm helium). $* p<0.05, * * p<0.01, * * * p<0.001$, $* * * * p<0.0001$ compared with the respective sham. (D) Xenon (50\% atm) (red bars) prevents injury developing after exposure to $55 \mathrm{kPa}$ shockwave. Xenon-treated blast-TBI slices (red bars) have reduced injury development compared with control slices treated with helium (blue bars). Xenon-treated blast-TBI slices were not significantly different from uninjured sham slices (white bars). Data have been normalized to control blast at $72 \mathrm{~h}$ after injury. Bars represent mean values, and error bars are standard errors $(n=22$, sham; $n=11$, blast $+50 \%$ atm xenon; $n=12$, blast $+50 \%$ at helium). $* p<0.05, * * p<0.01, * * * p<0.001$; $* * * p<0.0001$ compared with control blast. He, helium; Xe, xenon. 


\section{Discussion}

We developed an in vitro model of blast-TBI using organotypic hippocampal slice cultures, based on a blunt-TBI model that we have used to investigate the neuroprotective efficacy and mechanism of action of a variety of inert gases including xenon. ${ }^{36}$ The in vitro blunt-TBI model was first described by Coburn and colleagues, ${ }^{34}$ who were the first to show xenon to be protective in that model. We modified the blunt-TBI model to include submersion of slices on tissue culture inserts inside polythene sample bags, allowing them to be exposed to shockwaves from a horizontal gasdriven shock tube maintaining aseptic conditions.

Our blast model has some similarities to in vitro blast-TBI models described by Miller and coworkers ${ }^{41}$ and Effgen and associates. ${ }^{42}$ In our in vitro blast-TBI model, we observed an injury that increased with the magnitude of the peak overpressure and which exhibited a progressive injury developing over the course of $72 \mathrm{~h}$. This developing injury is similar to that observed in the in vitro blunt-TBI model ${ }^{34,36}$ and qualitatively similar to that observed in the blast-TBI models of Miller and coworkers ${ }^{41}$ and Effgen and associates. ${ }^{42}$ Our results are similar to those of Miller and coworkers ${ }^{41}$ who observed increases in PI fluorescence as early as $2 \mathrm{~h}$ after injury that developed up to $24 \mathrm{~h}$, using shockwaves of $147 \mathrm{kPa}$ POP, $10.4 \mathrm{kPa} \bullet \mathrm{msec}$ impulse, and $278 \mathrm{kPa}$ POP, $18.1 \mathrm{kPa} \bullet \mathrm{msec}$ impulse.

There are some quantitative differences between our model and that of Effgen and colleagues. ${ }^{42} \mathrm{We}$ observe blast-induced cell death at POP of $55 \mathrm{kPa}$ (impulse $10.3 \mathrm{kPa} \bullet \mathrm{msec}$ ) that is lower than the injury threshold for cell death observed by Effgen of $424 \mathrm{kPa}$ POP, $248 \mathrm{kPa} \bullet \mathrm{msec}$ impulse. ${ }^{43,44}$ Interestingly, Vogel, Effgen, and colleagues ${ }^{44}$ observed changes in the electrophysiological properties of slices at impulse levels as low as $\sim 39 \mathrm{kPa} \bullet \mathrm{msec}$, which are closer to the impulse levels in our experiments.

In our model, we observe blast-induced cell death significantly greater than sham levels at time points as early as $24 \mathrm{~h}$ with development up to $72 \mathrm{~h}$, whereas in the model of Effgen and coworkers, ${ }^{42}$ injury does not develop above sham level until $96 \mathrm{~h}$ after blast-injury. These differences may reflect the fact that our model used slices in a sterile bag while their model used a liquid filled receiver under a vertical shock tube, or the fact that we measured injury over the entire area of the slice while Effgen and coworkers ${ }^{42}$ used an approach based on region of interest, which may be less sensitive to a diffuse injury, such as the one caused by the exposure of OHSCs to a shockwave. We observed that PI fluorescence overlapped with cleaved caspase-3 immunostaining in blastexposed slices but not sham slices, indicating that the blastassociated cell death involves activation of apoptotic pathways.

\section{Xenon neuroprotection}

Treatment of the slices with $50 \%$ atm xenon, beginning $1 \mathrm{~h}$ after blast injury, caused a reduction in the degree of injury (cell death) at all time points tested, with xenon treatment reducing injury by between $31 \%$ and $47 \%$. The degree of protection against blast injury that we observed with $50 \%$ xenon is similar to that observed in the in vitro blunt-TBI model. ${ }^{34,36}$ At the $24 \mathrm{~h}$ and $72 \mathrm{~h}$ time points, the xenon-treated injured slices were not significantly different from the uninjured sham slices, consistent with xenon preventing injury development after blast-TBI in vitro.

The finding that xenon prevents blast injury developing up to $72 \mathrm{~h}$ after trauma is novel and is relevant to the potential clinical use of xenon, because clinical TBI lesions may develop significantly in the first $24 \mathrm{~h}$ after injury. ${ }^{45,46}$ The degree of protection that we observed with xenon is notable given that in our in vitro experimental protocol, xenon treatment only begins $1 \mathrm{~h}$ after injury, to simulate a clinically relevant time interval before medical care arrives after the blast injury. This is consistent with the time window for xenon neuroprotection observed in in vitro ${ }^{34}$ and in vivo ${ }^{31}$ models of blunt TBI and is relevant for clinical translation. We observed the greatest effect of xenon treatment on injury development within the first $24 \mathrm{~h}$, suggesting that treatment duration of $24 \mathrm{~h}$ or less may be effective. We have shown in an animal model of blunt TBI that xenon treatment for only three hours results in significant neuroprotection. ${ }^{31}$

\section{Limitations of the study}

Organotypic slice cultures are more complex than simple dissociated cell cultures, retain a heterogeneous population of cell types whose synaptic connectivity mirrors that seen in vivo, ${ }^{47-49}$ and are a useful tool to evaluate efficacy and mechanism of novel neuroprotective treatments. ${ }^{35,36}$ They cannot replace animal models to investigate injury development and neuroprotection, however. The use of a shock tube to generate Friedlander waves allows the isolated brain tissue to be subject to mechanical loading of short duration $(\sim 0.4 \mathrm{msec})$ modelling primary blast injury. Our in vitro model, however, cannot incorporate all of the other features of a real blast exposure in vivo, such as blunt injury (secondary blast), accelleration/deceleration (tertiary blast), or heat (quaternary blast). Nevertheless, in vitro models such as ours are a useful step in the translational process from bench to clinic.

\section{Conclusion}

The current study describes a novel in vitro model of blast-TBI. We show that injury magnitude depends on the intensity of the shockwave and that exposure to blast initiates caspase-dependent cell death. We demonstrate for the first time that treatment with xenon, starting $1 \mathrm{~h}$ after trauma, significantly limits injury progression after blast-induced TBI in vitro. Xenon could be delivered easily by inhalation shortly after brain injury with relatively simple equipment. In addition to its potential for arresting injury development, xenon has an additional advantage of simultaneously providing analgesia. ${ }^{50,51}$ Our findings support the idea that xenon may be a potential treatment for those with blast-induced TBI, and further research in this area is merited.

\section{Acknowledgments}

We thank Dr Amarjit Samra, Director of Research, Royal Centre for Defence Medicine, Birmingham, United Kingdom, for supporting this work, Scott Armstrong, Department of Surgery \& Cancer, Imperial College London, for assistance with preliminary experiments, Theofano Eftaxiopolou, Hari Arora, and Luz Ngoc Nguyen, Department of Bioengineering Imperial College London, and William Proud, Department of Physics Imperial College London, for advice on the shock-tube, Raquel Yustos, research technician, Department of Life Sciences, Imperial College London, for technical support, Neal Powell of the Department of Physics, Imperial College London, for artwork, Paul Brown, MBE, workshop manager, and Steve Nelson, workshop technician, Department of Physics, Imperial College London, for building the xenon chamber.

Study is supported by: Royal Centre for Defence Medicine, Birmingham, United Kingdom, Royal British Legion Centre for Blast Injury Studies, Imperial College London, United Kingdom. 
Medical Research Council, London, United Kingdom (MC_PC_ 13064; MR/N027736/1).

\section{Author Disclosure Statement}

Rita Campos-Pires was the recipient of a doctoral training award from the Fundação para a Ciência e a Tecnologia, Lisbon, Portugal. Mariia Koziakova was the recipient of a Rector's PhD Studentship from Imperial College London. Katie Harris was the recipient of a PhD studentship from the Westminster Medical School Research Trust, London, United Kingdom. Nicholas Franks has disclosed being a named inventor on a number of patents relating to the use of xenon as a neuroprotectant. He is a nonexecutive director of Neuroprotexeon Ltd. and has a financial interest in the use of xenon as a neuroprotectant. For the remaining authors, no competing financial interests exist.

\section{References}

1. Faul, M., Xu, L., Wald, M.M., and Coronado, V.G. Traumatic Brain Injury in the United States: Emergency Department Visits, Hospitalizations and Deaths 2002-2006. (2010). Atlanta, GA: Centers for Disease Control and Prevention, National Center for Injury Prevention and Control.

2. Warden, D. (2006). Military TBI during the Iraq and Afghanistan wars. J. Head Trauma Rehabil. 21, 398-402.

3. Independent Review Group. T.D. West and J.O. Marsh (eds). (2007). Alexandria VA: Independent Review Group.

4. Shively, S.B., Horkayne-Szakaly, I., Jones, R.V., Kelly, J.P., Armstrong, R.C., and Perl, D.P. (2016). Characterisation of interface astroglial scarring in the human brain after blast exposure: a post-mortem case series. Lancet Neurol. 15, 944-953.

5. Bell, R.S., Vo, A.H., Neal, C.J., Tigno, J., Roberts, R., Mossop, C., Dunne, J.R., and Armonda, R.A. (2009). Military traumatic brain and spinal column injury: a 5-year study of the impact blast and other military grade weaponry on the central nervous system. J. Trauma 66, S104-S111.

6. Owens, B.D., Kragh, J.F., Jr., Wenke, J.C., Macaitis, J., Wade, C.E., and Holcomb, J.B. (2008). Combat wounds in operation Iraqi Freedom and operation Enduring Freedom. J. Trauma. 64, 295-299.

7. Ahmed, F.A., Kamnaksh, A., Kovesdi, E., Long, J.B., and Agoston, D.V. (2013). Long-term consequences of single and multiple mild blast exposure on select physiological parameters and blood-based biomarkers. Electrophoresis 34, 2229-2233.

8. Kovesdi, E., Kamnaksh, A., Wingo, D., Ahmed, F., Grunberg, N.E., Long, J.B., Kasper, C.E., and Agoston, D.V. (2012). Acute minocycline treatment mitigates the symptoms of mild blast-induced traumatic brain injury. Front. Neurol. 3, 111.

9. Huber, B.R., Meabon, J.S., Martin, T.J., Mourad, P.D., Bennett, R., Kraemer, B.C., Cernak, I., Petrie, E.C., Emery, M.J., Swenson, E.R., Mayer, C., Mehic, E., Peskind, E.R., and Cook, D.G. (2013). Blast exposure causes early and persistent aberrant phospho- and cleavedtau expression in a murine model of mild blast-induced traumatic brain injury. J. Alzheimers Dis. 37, 309-323.

10. Logsdon, A.F., Lucke-Wold, B.P., Nguyen, L., Matsumoto, R.R., Turner, R.C., Rosen, C.L., and Huber, J.D. (2016). Salubrinal reduces oxidative stress, neuroinflammation and impulsive-like behavior in a rodent model of traumatic brain injury. Brain Res. 1643, 140-151.

11. Tweedie, D., Rachmany, L., Rubovitch, V., Li, Y., Holloway, H.W., Lehrmann, E., Zhang, Y., Becker, K.G., Perez, E., Hoffer, B.J., Pick, C.G., and Greig, N.H., (2016). Blast traumatic brain injury-induced cognitive deficits are attenuated by preinjury or postinjury treatment with the glucagon-like peptide-1 receptor agonist, exendin-4. Alzheimers Dement. 12, 34-48.

12. Pittinger, C.B., Moyers, J., Cullen, S.C., Featherstone, R.M., and Gross, E.G., (1953). Clinicopathologic studies associated with xenon anesthesia. Anesthesiology 14, 10-17.

13. Cullen, S.C. and Gross, E.G., (1951). The anesthetic properties of xenon in animals and human beings, with additional observations on krypton. Science 113, 580-582.

14. Ma, D., Hossain, M., Chow, A., Arshad, M., Battson, R.M., Sanders, R.D., Mehmet, H., Edwards, A.D., Franks, N.P., and Maze, M. (2005).
Xenon and hypothermia combine to provide neuroprotection from neonatal asphyxia. Ann. Neurol. 58, 182-193.

15. Homi, H.M., Yokoo, N., Ma, D., Warner, D.S., Franks, N.P., Maze, M., and Grocott, H.P. (2003). The neuroprotective effect of xenon administration during transient middle cerebral artery occlusion in mice. Anesthesiology 99, 876-881.

16. Martin, J.L., Ma, D., Hossain, M., Xu, J., Sanders, R.D., Franks, N.P., and Maze, M. (2007). Asynchronous administration of xenon and hypothermia significantly reduces brain infarction in the neonatal rat. Br. J. Anaesth. 98, 236-240.

17. Ma, D., Yang, H., Lynch, J., Franks, N.P., Maze, M., and Grocott, H.P. (2003). Xenon attenuates cardiopulmonary bypass-induced neurologic and neurocognitive dysfunction in the rat. Anesthesiology 98, 690698.

18. Dickinson, R. and Franks, N.P. (2010). Bench-to-bedside review: Molecular pharmacology and clinical use of inert gases in anesthesia and neuroprotection. Crit. Care 14, 229.

19. Dingley, J., Tooley, J., Porter, H., and Thoresen, M. (2006). Xenon provides short-term neuroprotection in neonatal rats when administered after hypoxia-ischemia. Stroke 37, 501-506.

20. Thoresen, M., Hobbs, C.E., Wood, T., Chakkarapani, E., and Dingley, J. (2009). Cooling combined with immediate or delayed xenon inhalation provides equivalent long-term neuroprotection after neonatal hypoxia-ischemia. J. Cereb. Blood Flow Metab. 29, 707-714.

21. Abraini, J.H., David, H.N., and Lemaire, M. (2005). Potentially neuroprotective and therapeutic properties of nitrous oxide and xenon. Ann. N. Y. Acad. Sci. 1053, 289-300.

22. Fries, M., Nolte, K.W., Coburn, M., Rex, S., Timper, A., Kottmann, K., Siepmann, K., Hausler, M., Weis, J., and Rossaint, R. (2008). Xenon reduces neurohistopathological damage and improves the early neurological deficit after cardiac arrest in pigs. Crit. Care Med. 36, 2420-2426.

23. Sheng, S.P., Lei, B., James, M.L., Lascola, C.D., Venkatraman, T.N., Jung, J.Y., Maze, M., Franks, N.P., Pearlstein, R.D., Sheng, H., and Warner, D.S. (2012). Xenon neuroprotection in experimental stroke: interactions with hypothermia and intracerebral hemorrhage. Anesthesiology 117, 1262-1275.

24. Armstrong, S.P., Banks, P.J., McKitrick, T.J., Geldart, C.H., Edge, C.J., Babla, R., Simillis, C., Franks, N.P., and Dickinson, R. (2012). Identification of two mutations (F758W and F758Y) in the N-methylD-aspartate receptor glycine-binding site that selectively prevent competitive inhibition by xenon without affecting glycine binding. Anesthesiology 117, 38-47.

25. Dickinson, R., Peterson, B.K., Banks, P., Simillis, C., Martin, J.C., Valenzuela, C.A., Maze, M., and Franks, N.P. (2007). Competitive inhibition at the glycine site of the N-methyl-D-aspartate receptor by the anesthetics xenon and isoflurane: evidence from molecular modeling and electrophysiology. Anesthesiology 107, 756-767.

26. Franks, N.P., Dickinson, R., de Sousa, S.L., Hall, A.C., and Lieb, W.R. (1998). How does xenon produce anaesthesia? Nature 396, 324.

27. Gruss, M., Bushell, T.J., Bright, D.P., Lieb, W.R., Mathie, A., and Franks, N.P. (2004). Two-pore-domain K+ channels are a novel target for the anesthetic gases xenon, nitrous oxide, and cyclopropane. Mol. Pharmacol. 65, 443-452.

28. Bantel, C., Maze, M., and Trapp, S. (2010). Noble gas xenon is a novel adenosine triphosphate-sensitive potassium channel opener. Anesthesiology 112, 623-630.

29. Shu, Y., Patel, S.M., Pac-Soo, C., Fidalgo, A.R., Wan, Y., Maze, M., and Ma, D. (2010). Xenon pretreatment attenuates anesthetic-induced apoptosis in the developing brain in comparison with nitrous oxide and hypoxia. Anesthesiology 113, 360-368.

30. Laitio, R., Hynninen, M., Arola, O., Virtanen, S., Parkkola, R., Saunavaara, J., Roine, R.O., Gronlund, J., Ylikoski, E., Wennervirta, J., Backlund, M., Silvasti, P., Nukarinen, E., Tiainen, M., Saraste, A., Pietila, M., Airaksinen, J., Valanne, L., Martola, J., Silvennoinen, H., Scheinin, H., Harjola, V.P., Niiranen, J., Korpi, K., Varpula, M., Inkinen, O., Olkkola, K.T., Maze, M., Vahlberg, T., and Laitio, T. (2016). Effect of inhaled xenon on cerebral white matter damage in comatose survivors of out-of-hospital cardiac arrest: a randomized clinical trial. JAMA. 315, 1120-1128.

31. Campos-Pires, R., Armstrong, S.P., Sebastiani, A., Luh, C., Gruss, M., Radyushkin, K., Hirnet, T., Werner, C., Engelhard, K., Franks, N.P., Thal, S.C., and Dickinson, R. (2015). Xenon improves neurologic outcome and reduces secondary injury following trauma in an in vivo model of traumatic brain injury. Crit. Care Med. 43, 149-158. 
32. Campos-Pires, R. and Dickinson, R. Modelling Blast Brain Injury, in: Blast Injury Science \& Engineering: A guide for Clinicians \& Researchers. A. Bull, J. Clasper, and P. Mahoney (eds). (2016). New York: Springer International. pp. 173-182.

33. Stoppini, L., Buchs, P.A., and Muller, D., (1991). A simple method for organotypic cultures of nervous tissue. J. Neurosci. Methods 37, 173-182.

34. Coburn, M., Maze, M., and Franks, N.P. (2008). The neuroprotective effects of xenon and helium in an in vitro model of traumatic brain injury. Crit. Care Med. 36, 588-595.

35. Banks, P., Franks, N.P., and Dickinson, R. (2010). Competitive inhibition at the glycine site of the N-methyl-D-aspartate receptor mediates xenon neuroprotection against hypoxia-ischemia. Anesthesiology 112, 614-622.

36. Harris, K., Armstrong, S.P., Campos-Pires, R., Kiru, L., Franks, N.P., and Dickinson, R. (2013). Neuroprotection against traumatic brain injury by xenon, but not argon, is mediated by inhibition at the N-methyl-D-aspartate receptor glycine site. Anesthesiology 119 , 1137-1148.

37. Macklis, J.D. and Madison, R.D. (1990). Progressive incorporation of propidium iodide in cultured mouse neurons correlates with declining electrophysiological status: a fluorescence scale of membrane integrity. J. Neurosci. Methods 31, 43-46.

38. Noraberg, J., Kristensen, B.W., and Zimmer, J. (1999). Markers for neuronal degeneration in organotypic slice cultures. Brain Res. Brain Res. Protoc. 3, 278-290.

39. Vornov, J.J., Tasker, R.C., and Coyle, J.T. (1991). Direct observation of the agonist-specific regional vulnerability to glutamate, NMDA, and kainate neurotoxicity in organotypic hippocampal cultures. Exp. Neurol. 114, 11-22.

40. Schneider, C.A., Rasband, W.S., and Eliceiri, K.W. (2012). NIH Image to ImageJ: 25 years of image analysis. Nat. Methods 9, 671-675.

41. Miller, A.P., Shah, A.S., Aperi, B.V., Budde, M.D., Pintar, F.A., Tarima, S., Kurpad, S.N., Stemper, B.D., and Glavaski-Joksimovic, A. (2015). Effects of blast overpressure on neurons and glial cells in rat organotypic hippocampal slice cultures. Front. Neurol. 6, 20.

42. Effgen, G.B., Hue, C.D., Vogel, E. 3rd, Panzer, M.B., Meaney, D.F., Bass, C.R., and Morrison, B. 3rd (2012). A multiscale approach to blast neurotrauma modeling: Part II: methodology for inducing blast injury to in vitro models. Front. Neurol. 3, 23.

43. Effgen, G.B., Vogel, E.W., 3rd, Lynch, K.A., Lobel, A., Hue, C.D., Meaney, D.F., Bass, C.R., and Morrison, B. 3rd. (2014). Isolated primary blast alters neuronal function with minimal cell death in organotypic hippocampal slice cultures. J. Neurotrauma. 31, 1202-1210.

44. Vogel, E.W. 3rd, Effgen, G.B., Patel, T.P., Meaney, D.F., Bass, C.R., and Morrison, B., 3rd. (2016). Isolated primary blast inhibits longterm potentiation in organotypic hippocampal slice cultures. J. Neurotrauma 33, 652-661.

45. Servadei, F., Nanni, A., Nasi, M.T., Zappi, D., Vergoni, G., Giuliani, G., and Arista, A. (1995). Evolving brain lesions in the first 12 hours after head injury: analysis of 37 comatose patients Neurosurgery 37 , 899-906.

46. White, C.L., Griffith, S., and Caron, J.L. (2009). Early progression of traumatic cerebral contusions: characterization and risk factors. J. Trauma 67, 508-514.

47. Bahr, B.A. (1995). Long-term hippocampal slices: a model system for investigating synaptic mechanisms and pathologic processes. J. Neurosci. Res. 42, 294-305.

48. Crain, S.M. (1998). Development of specific synaptic network functions in organotypic central nervous system (CNS) cultures: implications for transplantation of CNS neural cells in vivo. Methods 16 , 228-238.

49. Finley, M., Fairman, D., Liu, D., Li, P., Wood, A., and Cho, S. (2004). Functional validation of adult hippocampal organotypic cultures as an in vitro model of brain injury. Brain Res. 1001, 125-132.

50. Petersen-Felix, S., Luginbuhl, M., Schnider, T.W., Curatolo, M., Arendt-Nielsen, L., and Zbinden, A.M. (1998). Comparison of the analgesic potency of xenon and nitrous oxide in humans evaluated by experimental pain. Br. J. Anaesth. 81, 742-747.

51. Giacalone, M., Abramo, A., Giunta, F., and Forfori, F. (2013). Xenonrelated analgesia: a new target for pain treatment. Clin. J. Pain 29, 639-643.

Address correspondence to:

Robert Dickinson, PhD

Sir Ernst Chain Building

Imperial College London

South Kensington, London SW7 2AZ

United Kingdom

E-mail: r.dickinson@imperial.ac.uk 